\title{
Harnessing the potential clinical use of medicinal plants as anti-diabetic agents
}

\author{
This article was published in the following Dove Press journal: \\ Botanics: Targets and Therapy \\ 2I August 2012 \\ Number of times this article has been viewed
}

\author{
Joan IA Campbell-Tofte' \\ Per Mølgaard ${ }^{2}$ \\ Kaj Winther' \\ 'Department of Clinical Biochemistry, \\ Frederiksberg University Hospital, \\ Frederiksberg, Denmark; ${ }^{2}$ Department \\ of Medicinal Chemistry, Faculty of \\ Pharmaceutical Sciences, University of \\ Copenhagen, Copenhagen, Denmark
}

Correspondence: Joan Campbell-Tofte Department of Clinical Biochemistry, Coordinating Research Unit, Frederiksberg University Hospital, Nordre Fasanvej 57, 2000 Frederiksberg, Denmark $\mathrm{Tel}+4538 \quad 163448$ Fax $+45381647 \quad 19$

Email joan.campbell-tofte@frh.regionh.dk
Abstract: Diabetes is a metabolic disorder arising from complex interactions between multiple genetic and/or environmental factors. The characteristic high blood sugar levels result from either lack of the hormone insulin (type 1 diabetes, T1D), or because body tissues do not respond to the hormone (type 2 diabetes, T2D). T1D patients currently need exogenous insulin for life, while for T2D patients who do not respond to diet and exercise regimes, oral anti-diabetic drugs (OADs) and sometimes insulin are administered to help keep their blood glucose as normal as possible. As neither the administration of insulin nor OADs is curative, many patients develop tissue degenerative processes that result in life-threatening diabetes comorbidities. Several surveys of medicinal plants used as anti-diabetic agents amongst different peoples have been published. Some of this interest is driven by the ongoing diabetes pandemic coupled with the inadequacies associated with the current state of-the-art care and management of the syndrome. However, there is a huge cleft between traditional medicine and modern (Western) medicine, with the latter understandably demanding meaningful and scientific validation of anecdotal evidence for acceptance of the former. The main problems for clinical evaluation of medicinal plants with promising anti-diabetic properties reside both with the complexity of components of the plant materials and with the lack of full understanding of the diabetes disease etiology. This review is therefore focused on why research activities involving an integration of Systems Biology-based technologies of pharmacogenomics, metabolomics, and bioinformatics with standard clinical data, should be used for cost-effective validation of the safety and anti-diabetic efficacy of promising medicinal plants. The application of such approaches to studying entire mixtures of plant materials will ensure proper elucidation of novel therapies with improved mechanisms of action, as well as facilitate a personalized clinical use of medicinal plants as anti-diabetic agents.

Keywords: medicinal plants, anti-diabetes therapy, natural products, systems biology, 'omics technologies, drug discovery

\section{Introduction}

With the current emphasis on finding lead compounds for specific diseases through rational protein-targeted, structure-based designs, high-throughput bioassay-based screening, and virtual screenings, it is easy to forget that metformin has its origins in the use of the humble French lilac in European folk medicine (Galega officinalis, Fam: Fabaceae). ${ }^{1}$ The inspiration for the synthesis of biguanides like metformin, came from a guanidine derivative - galegine, an active component of Galega officinalis. ${ }^{2}$ Metformin is currently the first-line drug of choice recommended for managing type 2 diabetes (T2D), by both the American Diabetes Association and European Association for the Study of Diabetes. ${ }^{3}$ In this review we explore the possibilities for finding innovative 
and more effective treatments for curbing the current scourge of diabetes, by mining the therapeutic potentials in other medicinal plants used in traditional medicine.

Diabetes mellitus is a metabolic disorder which arises from complex interactions between multiple genetic and environmental or lifestyle factors. This chronic disease is characterized by the presence of hyperglycemia due to defective insulin secretion, insulin action, or both. Long-term diabetes is associated with several comorbidities, such as erectile dysfunction, blindness, poor wound healing, kidney failure, heart disease, etc; as a result of considerable damage, dysfunction, and failure of various organs that develop as the disease progresses.

The incidence of diabetes worldwide is now estimated to be around 366 million, far beyond the 285 million projected by WHO for 2010 from global statistics gathered in 2008. ${ }^{4}$ This means that there may have been more than 4 million deaths or $6.8 \%$ of global mortality in 2010 that could be attributed directly or indirectly to diabetes, ${ }^{5}$ and explains why global diabetes health expenditure in 2010 was estimated at $€ 263$ billion or $12 \%$ of total global health care costs. ${ }^{6}$ The two major forms of the syndrome result from either lack of the metabolism regulatory hormone, insulin (type 1 diabetes, T1D), or because body tissues fail to respond to the hormone (T2D). The majority or $90 \%$ of patients with diabetes have T2D. As insulin is crucial for the maintenance of life, T1D patients depend on externally administered insulin, while for T2D patients who do not respond to diet and exercise regimes, oral anti-diabetes drugs (OADs) and sometimes external insulin are administered to help keep their blood glucose as normal as possible. According to the data from WHO, the highest increases in diabetes prevalence are amongst low- and middle-income countries, predominantly within the 40-59 years age group, although a tendency is seen for onset at a younger age. ${ }^{7}$ Thus, apart from the astronomical global health care costs involved in treating and managing diabetes, this disease imposes additional social economic burdens from lost productivity and foregone economic growth. Therefore, there is a need for the development of more effective preventive solutions and therapeutic approaches that address and abolish the reduction in life expectancy and life quality imposed by diabetes and its complications or comorbidities.

While obesity and lack of exercise are the primary environmental factors known to be closely associated with the global T2D upsurge, advances in genome wide screenings have only revealed a few genes that increase the risk of developing the illness. For example, the gene
$I F I H 1$, which codes for interferon induced with helicase C domain 1, has been implicated in the etiology of T1D. ${ }^{8}$ Some variants of the Beta3-adrenergic receptor gene, ${ }^{9}$ peroxisome proliferator-activated receptor- (PPARgamma) gene in both Caucasian ${ }^{10}$ and Chinese ${ }^{11}$ patients, PPARgamma co-activator 1 -alpha gene $(P C G-1),{ }^{12}$ and the gene coding for the T-cell specific transcription factor TCF7L2, ${ }^{13}$ have been linked to higher risks of developing T2D. However, it is not clear how environmental factors interact with the gene products to cause the disease. The current understanding of the pathophysiology of T2D is that several factors referred to as the 'ominous octet' underlie the disease development and progression into tissue degenerations and the diabetic complications like cardiovascular diseases, amputation, poor wound healing, etc. ${ }^{14}$ The components of the ominous octet include: pancreatic $\alpha$-cell and $\beta$-cell dysfunction, increased hepatic glucose production, decreased insulin-sensitivity in muscle, adipocytes and central nervous system, reduced gastrointestinal incretin secretion/sensitivity and enhanced glucose reabsorption in kidneys ${ }^{14}$

\section{Current diabetes therapy and associated problems}

According to the road map for diabetes research that was recently commissioned by the European Research Council (DIAMAP), ${ }^{15}$ the status of current anti-diabetes treatment can be summarized as follows:

'Today, there is no cure for this disease; while new drugs
and a holistic approach to treatment have certainly improved
the prognosis for individuals with diabetes and their quality
of life, the menace, morbidity and increased mortality from
micro- and macro-vascular complications remain.'

Similarly, the latest statement of the American Diabetes Association's Standards of Medical Care in Diabetes ${ }^{16}$ states that:

'Diabetes is a chronic illness that requires continuing medical care and on-going patient self-management education and support to prevent acute complications and to reduce the risk of long-term complications. Diabetes care is complex and requires that many issues, beyond glycemic control, be addressed.'

As diabetes is commonly regarded as a disorder involving chronically elevated blood sugar levels, anti-diabetic therapy has to a large extent been 'glucose fixated'. This means that the therapeutic agents that are currently used to treat or 
manage diabetes work directly or indirectly by regularizing/ normalizing blood glucose levels. They include:

1. Insulin for T1D patients who lack the hormone, and for T2D patients who exhibit poor blood glucose management and are unable to meet or keep within safe glycemic goals.

2. Agents that increase the amount of insulin secreted by the pancreas - sulfonylureas (eg, amaryl marketed by Sanofi-Aventis, Paris, France) and new generation incretins and glucagon-like peptide analogs and agonists; (eg, tolbutamide, glimepiride; and the newer exenatide [Amylin Pharmaceuticals, San Diego, CA and Eli Lilly and Company, Indianapolis, IN)], liraglutide [Novo Nordisk, Bagsværd, Denmark, etc]).

3. Agents which increase the sensitivity of target tissues like skeletal muscle to insulin, eg, biguanides and thiazolidinediones; (includes metformin and pioglitazone).

4. Agents that decrease the rate at which glucose is absorbed from the gastrointestinal tract, (eg, acarbose and miglitol). Alpha-glucosidase inhibition of alpha-amylase in the gut by foods and nutritional supplements also decrease glucose uptake.

5. Bariatric surgery for adults with BMI $>35 \mathrm{~kg} / \mathrm{m}^{2}$ and T2D, especially where the diabetes or associated comorbidities are difficult to control with lifestyle and pharmacologic interventions.

The choice of anti-diabetic therapy is therefore highly dependent on the clinical assessment of the individual patients. All the above measures serve their purpose to some extent, and apart from bariatric surgery (as indicated in small trials), ${ }^{17}$ the disease is neither cured nor is the development of the adverse complications associated with long-term diabetes prevented. However, bariatric surgery is costly in the short term and has some serious risks as it is highly invasive and requires dramatic changes in lifestyles; eg, the need to eat only fluid food. Finally, the procedure is not yet available to patients who are not severely obese because the mechanisms of diabetes remission (ie, blood glucose normalization without medications) and the long-term benefits and risks are yet to be properly defined.

\section{Medicinal plants in diabetes care and management}

Much of the world's population depends on plant materials for food and life sustenance. Given the proximity and availability of plants and humans in their common evolution, it is not surprising that many cultures have also used plant materials when seeking solutions to different ailments. The WHO fact sheet No 134 estimates that $80 \%$ of the population in Asia and Africa depend on traditional medicine (of which herbal medicines are the major part), for their primary health care. ${ }^{18}$

There are four basic ways in which medicinal plants that are used in traditional medicine become valuable tools in modern medicine: ${ }^{19}$

- As direct sources of therapeutic agents in the form of plant powders, extracts, or phytochemicals (eg, the alkaloid D-tubocurarine, a skeletal muscle relaxant that was used adjunctively in anesthesia, was extracted from the South American jungle plant Chondrodendron tomentosum). ${ }^{20}$

- As starting points or building blocks of semi-synthetic compounds.

- As models for new synthetic compounds. Cocaine from the Coca plant, Erythroxylum coca, has served as a model for the synthesis of a number of local anesthetics such as procaine. ${ }^{21}$

- As taxonomic markers for the discovery of new compounds. From a phytochemical standpoint, other species of the same family may contain more and perhaps, better forms of the desired end products.

According to the definitions in the opening editorial of Botanics: Targets and Therapy, botanical pharmaceutical preparations are used either as plant materials or in the form of phytochemicals. ${ }^{22}$ Herbal or plant materials are standardized herbal pharmaceutical preparations consisting of complex mixtures of one or more plants which contain plant parts or plant extracts in the crude or processed state as active ingredients, while phytochemicals are selected fractions of the plant extracts with health-related bioactivity.

\section{Plants with potential anti-diabetic activity}

Plants are very smart biochemists, making over 200,000 unique secondary metabolites in both food and non-food plant materials. The terminology nutraceutical is often used to distinguish between the plant-derived compounds isolated or purified from foods and generally sold in medicinal forms; from the herbal pharmaceutical preparations which originate in non-food plant materials. In this review, a distinction was not made between the two terminologies, except to acknowledge that particular attention to foods naturally rich in nutraceuticals with proven anti-diabetic properties, or artificially enriched with such compounds, as is the case in 'functional foods', will definitely help with improving glycemic control and in delaying the development of diabetic complications. Examples of such a scenario can be found in 
a review by Srinivasan, who outlined the scientific basis for the beneficial physiological effects of most of the commonly used spices in Indian cooking. ${ }^{23}$

Extensive surveys and reviews on ethnobotanical information and scientific investigations of medicinal plants used for treating diseases with diabetes-like symptoms abound in the literature as summarized in Table 1. The list is by no means exhaustive and is only included as a guide to researchers and practitioners. In a systematic review of the published literature on the efficacy and safety of herbal therapies and vitamin/mineral supplements for glucose control, Yeh et al observed that a majority of the plant-sourced medicines appear to be safe in the doses administered. ${ }^{24}$ However, for most of the concoctions, there is often insufficient evidence to draw definitive conclusions about their efficacy. The few herbals with evidence for anti-diabetic efficacy from adequately designed randomized and placebo-controlled trials include Coccinia indica, ${ }^{25}$ American ginseng (Panax quinquefolius L.), ${ }^{26}$ Cinnamon, ${ }^{27}$ (tree bark of the species Cinnamomum sp.) and the Rauvolfia-Citrus decoction. ${ }^{28}$

Within the last two decades, there has been a renaissance of interest in Western countries for treatment solutions known in local traditional medicine and considerable adoption of foreign traditional medicinal practices, particularly of Chinese and Ayurvedic origins. ${ }^{19}$ Whether this phenomenon is driven by increased immigration or globalization, the
WHO fact sheet No 134 also states that $70 \%-80 \%$ of the population in developed countries has used some form of alternative or complementary medicine. ${ }^{18}$ Herbal treatments are the most popular form of traditional medicine because of the relative ease by which they are administered, as compared for example, to acupuncture which requires the services of trained personnel. Plant-derived traditional medicines have thus become very lucrative business in the international marketplace. Annual revenues reported for the sale of herbal medicines in Western Europe reached US\$5 billion in 2003-2004, as compared to US\$14 billion of same product sales in China in 2005. ${ }^{18}$

While recognizing the potential of traditional medicine for providing treatment solutions for various infectious and chronic diseases, as well as the temptation that comes with big unregulated business, WHO has called for urgency in setting up international standards and methods for the evaluation of safety and therapeutic efficacy of traditional medicinal approaches. Such measures should prevent the introduction of poor quality herbal products and facilitate the smooth introduction of traditional medicine practices into conventional medical practices. Herein lies an added challenge, as far as harnessing the potential of medicinal plants is concerned: the interest in adopting therapeutic agents from traditional medicine in the general population is indirectly proportional to the interest of practitioners of conventional medicine for

Table I Ethnobotanical surveys and reviews of medicinal plants with possible anti-diabetic activity

\begin{tabular}{|c|c|c|}
\hline Reference & Region covered & Validation/experimental methods \\
\hline Yeh et $\mathrm{al}^{24}$ & Published reports from all over the globe & Randomized and non-randomized controlled clinical trials \\
\hline Jia et $a^{68}$ & China & A Chinese database for medicinal plants with anti-diabetic activity \\
\hline Atawodi 69 & Africa & $\begin{array}{l}\text { Survey of medicinal plants with antioxidant potential of African } \\
\text { origin reported between } 1965 \text { and } 2004 \text {. Includes those } \\
\text { with anti-diabetic activity }\end{array}$ \\
\hline Gurib-Fakim $^{19}$ & India and Africa & $\begin{array}{l}\text { Provides an overview of some plants with well-known profiles } \\
\text { of anti-diabetic properties }\end{array}$ \\
\hline Bnouham et $\mathrm{al}^{70}$ & $\begin{array}{l}\text { Collated data on plants with hypoglycemic effects } \\
\text { from every region that was reported in MEDLINE } \\
\text { (PubMed) over 1990-2000 }\end{array}$ & Included only studies that tested plant extracts in pre-clinical tests \\
\hline Jung et $\mathrm{al}^{71}$ & $\begin{array}{l}\text { Included medicinal plants that have been used } \\
\text { in traditional medicine in different parts of the globe }\end{array}$ & Have reported experimental or clinical anti-diabetic activity \\
\hline Khalil et $\mathrm{al}^{72}$ & Brazil, (South America) & In vitro antioxidant assays \\
\hline Evans $^{73}$ & From all over the globe & Pre-clinical and clinical trials \\
\hline Afolayan and Sunmonu ${ }^{74}$ & Southern Africa & In vivo bioassays \\
\hline Begum et $\mathrm{al}^{75}$ & $\begin{array}{l}\text { Includes plant extracts and phytochemicals from } \\
\text { plant materials in Bangladesh, India, Pakistan, Nepal, } \\
\text { Sri Lanka, China, and Cameroon }\end{array}$ & $\begin{array}{l}\text { Screened anti-diabetic efficacy using non-diabetic, type I, } \\
\text { and type } 2 \text { diabetes animal models }\end{array}$ \\
\hline Kavishankar et $\mathrm{al}^{76}$ & $\begin{array}{l}\text { Plants with anti-diabetic and related beneficial } \\
\text { effects originating from different parts of world }\end{array}$ & Literature survey \\
\hline Patel et $\mathrm{al}^{77}$ & From everywhere & $\begin{array}{l}\text { Survey of bioactive drugs and isolated compounds from plants } \\
\text { showing significant insulin mimetic and anti-diabetic activity } \\
\text { with more efficacy than conventional hypoglycemic agents }\end{array}$ \\
\hline
\end{tabular}


procuring scientific evidence for the safety and effectiveness of traditional medicine products and practices.

\section{Reasons for poor acceptance of medicinal plants in conventional medicine}

The reasons for this disparity between the general public's interest in traditional medicine and the momentum for its incorporation into modern (conventional) medicine can be summarized as follows:

1. There is often a lack of scientific evidence for safety and efficacy for traditional medicines: most of the patients eager to take traditional medicine are usually suffering from chronic and complex diseases, for which conventional medicine neither has a cure nor a complete understanding. Hence, the absence of a clear understanding of the mechanisms of the alleged therapeutic effect has hindered the advancement of many a promising herbal medicine. Each product may contain several different plants, each plant contributing potentially hundreds of chemical constituents, some of which may be present in very low concentrations. Laboratory investigations of single components and/or every potential combination are both impractical and costly in terms of time and money. ${ }^{29}$ Given that the chemistry and efficacy of many of the components in the plant mixtures are relatively unknown, patients taking the treatments might be unwittingly exposing themselves to the risks of toxicity or overdose.

2. Poor regulations and quality controls: in the wake of the monetary gain to be had, it is not surprising that many of the reported side effects associated with the use of traditional medicines come from poor quality control in product manufacture. These can come from errors in plant identification, poor manufacturing practices, and/ or lack of product standardization, contamination of end products, substitution, or incorrect preparations or dosage. Hence, there is a need for proper regulation of both manufacture and use of herbal medicines.

3. Variation in source plant materials and resulting medicinal products: the constituents of traditional phytopreparations are often affected by environmental factors such as light, temperature, soil quality, period, and time of harvest, as well as the age of the plant..$^{30,31}$ These factors complicate the process of producing consistent medicinal products, let alone producing them in sufficient quantities for medicinal use or for testing purposes. ${ }^{32}$ Again, the natural and man-imposed variations in source plants highlight the need for proper product standardization in plant medicines.
4. False belief in the safety of herbals: there is an unfortunate false perception with many members of the general public that traditional medicinal products are safer because they are prepared from natural sources without much processing. ${ }^{33}$ In reality, plant-derived secondary metabolites are far from being benign molecules, especially considering that several of them were evolved as chemical defenses that can repel, stun, poison, or kill other species. ${ }^{19}$ As with conventional medicines, traditional medicines must be judiciously prescribed and used, such that there is a clear and unequivocal awareness of potential herb-herb and herb-drug interactions.

5. Lack of venture capital investors, high risk, and high costs: in comparison to conventional medicinal products where development typically takes an average of 12 years and costs hundreds of millions of US dollars/euros, ${ }^{34}$ subjecting the complex mixtures in phytopreparations to the same product development process would take significantly longer time periods and incur greater costs. As explained above, screening each of the components or combinations of the chemicals in the complex plant mixture as it is done for conventional medicines would be impractical and too expensive. Even with technological developments that render the process more cost effective, the researchers cannot take out proprietary rights over a tree or its fruit, as there is no way to prevent others from growing a particular plant for personal use. ${ }^{35}$ For fear of making small returns on their investments, venture capital is often not prepared to put up the amounts of money required for analyzing and standardizing herbal medicines.

In spite of these reservations, we believe that if the right scientific methods are applied, medicinal plants with already established anti-diabetic properties in traditional medicine may yet prove to be valuable resources for innovative, evidence-based, and effective treatment solutions for the disease.

\section{Verification of efficacy and safety of medicinal plants as anti-diabetic agents Identification and drug development strategies starting from plant materials}

State-of-the-art screening for bioactivity in natural products is a 'top down' approach, starting with the larger units, for example, whole plant material, and working down to the specific fractions or combinations responsible for the bioactivity 
of interest. Briefly, the source plant materials are collected and preprocessed for ease of obtaining the 'working mixture'. The 'working mixture' is used here to refer to the form administered for therapeutic purposes and can be in the form of powders, teas or infusions, decoction, juice, tincture, or poultice. Initial preparations of the working mixture for analyses involve concentration (for the liquid forms) or extraction with suitable solvents and concentration of the resulting extract (for the solid or viscous forms). The concentrated plant extract and fractionations there-of can then be tested in vivo as in preclinical animal tests or in controlled clinical trials; or in in vitro tests based on pre-defined bioassays for the evaluation of their bioactivity, as illustrated in Figure 1. Ideally, any therapeutic claims for either the complex mixtures of phytotherapies or isolated phytochemicals would be confirmed or refuted in randomized, placebo-controlled clinical trials.

There are several approaches to choosing source plant materials. With increasing knowledge of the molecular processes underlying disease states, and astronomical improvements in separation and compound identification technologies, many pharmaceutical companies embarked on randomly selecting source plants, followed by in vitro, mechanism-based, high throughput screening (HTS) in the initial phases of plant drug research (refer to $\mathrm{C}$ in Figure 1). The poor harvest from this random endeavor was the basis for

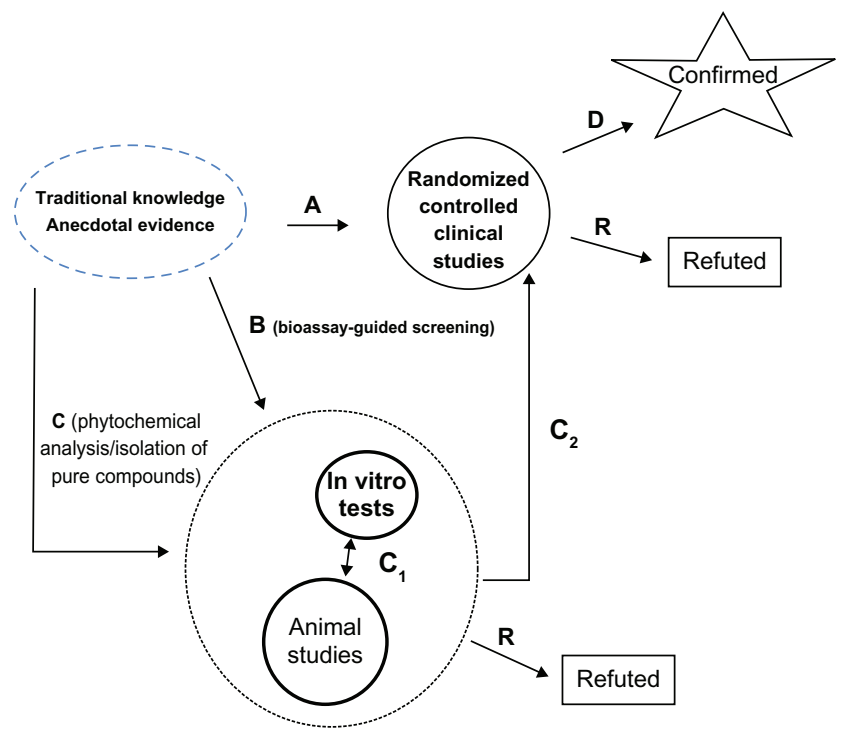

Figure I Idealized process for evaluating the efficacy of medicinal plant materials. Traditional herbal drugs can either be directly assessed in clinical trials $(\mathbf{A})$, or used as a basis for bio-prospecting (B), or subjected to pre-clinical animal experiments and in vitro studies related to their ethnopharmacological indications (B). Finally, the pharmacological effect of the plant extract is either refuted (R) or confirmed (D) in the clinical studies. Similarly, empirical data obtained from $\mathbf{B}$ and $\mathbf{C}$ are either refuted during the animal experiments and in vitro studies in $C_{1}$ or confirmed or refuted via $\mathrm{C}_{2}$ in randomized, double-blinded, placebo-controlled clinical studies. Reprinted from J Ethnopharmacol. 122, Gertsch J. How scientific is the science in ethnopharmacology? Historical perspectives and epistemological problems. I77-183. Copyright 2009. with permission from Elsevier. ${ }^{36}$ advocating source plant selection based on the ethnomedical approach. ${ }^{37}$ The argument for using ethnomedicinal use as basis of choice of starting material is that physicians of the ancient world utilized botanical medicines to treat diabetes. As such, a systematic and intensive search for new drugs to treat T2D amongst medicinal plants with a long history of human use would be practical, cost-effective, and logical. More recently, phylogenetic frameworks have served as a basis for the selection of plants for medicine and drug development, ${ }^{20}$ as evolutionarily-related medicinal plants may represent better sources (both in quality and quantity) of the desired bioactive agents.

\section{Current technical challenges with harnessing the potential in medicinal plants Dependency on in vitro bioassays}

However the starting material is chosen, the success of modern drug development programs is very dependent on which in vitro bioassays are used during HTS. In the in vitro bioassays, specific biological processes or reactions are set up outside the living system, such that the workings of individual proteins or pathways can be tested in a test tube. Examples of some of the in vitro bioassays involving molecular targets important for treating diabetes are outlined below. Advances in molecular biology and in automation have made it possible for researchers to design and carry out millions of such assays. Through this step, active compounds which modulate a particular aspect of a biomolecular pathway or the activity of a specific protein are rapidly identified to provide an understanding of the mechanisms of action or roles for individual components in biochemical processes. The earliest in vitro bioassays were more of 'the single hit' type, used for investigating compounds that might have antibacterial and antifungal activity. With increasing interest in finding therapeutic agents for complex syndromes like cancer, diabetes, and neurological diseases, came the necessity to avoid overpredictive claims of bioactivity and hence batteries of test systems replaced the single test. ${ }^{38}$ The diversification in bioassay systems brought substantial increases in the costs of the drug development process. In spite of the drawbacks inherent with in vitro tests, that is, their ineffectiveness for addressing issues of drug transport from the site of administration to the site of action, or issues of secondary metabolism after drug administration, the application of HTS has provided several successful 'hits' or relevant drug candidates in the past. 
However, a major drawback with HTS is that there is the absolute requirement for a target or reference logical entity (this could be a cell, protein, or an animal embryo) that the screening is conducted upon. In other words, drug discovery often begins with a known biological target.

Therefore, the well-characterized/established bioassay set-up emphasizes finding new drugs to known targets, but leaves no room for innovative treatments with hitherto unknown targets or mechanisms.

It is therefore not surprising that in the current glucosecentric environment of diabetes care and management, bioassays for anti-diabetic agents target compounds/treatment solutions that are hypoglycemic or modify one of the several proteins already implicated in the pathophysiology of diabetes. The rationales for some of the in vitro bioassays used for identifying agents useful for anti-diabetes therapy include:

A. Stimulation of insulin release from pancreatic cells presented as isolated islets of Langerhans ${ }^{39}$ or as cultured islet beta-cell lines. ${ }^{40}$ In this bioassay, insulin release into the culture filtrates is measured by radioimmunoassay and compared in samples collected before and after treating the cells with the test substances. Three out of 32 plant species used in Ayurvedic medicine for treating diabetes tested positive when subjected to this test. ${ }^{39}$

B. Inhibition of glucose absorption from the intestine is tested in bioassays designed to measure alpha-amylase or alphaglucosidase inhibitory activity. The rationale behind this approach is to treat diabetes using agents which inhibit these enzymes that convert complex dietary carbohydrates into absorbable sugar molecules. The resulting inhibition of glucose uptake should decrease postprandial hyperglycemia and the glucose stress diabetics are subjected to. The in vitro bioassay method is based on measuring the rate of sugar release. This concept was used to test lipophilic extracts of six Malaysian species, of which Phyllanthus amarus showed significant inhibition of alpha-amylase. The activity came primarily from the triterpenes - ursolic and oleanolic acids. ${ }^{41}$ Similarly, the inhibition of ß-glucosidase by extracts of Shorea robusta Gaertn. and Emblica officinalis Gaertn., was determined by measuring the amount of $p$-nitrophenol liberated from the substrate $p$-nitrophenyl- $\beta$-D-glucopyranoside. ${ }^{42}$

C. Promotion of glucose utilization in isolated tissues and cells, notably by adipocytes. A hot water extract of Lagerstroemia speciosa leaves was found to stimulate glucose uptake in cultured 3T3-L1 adipocytes. The observation was interpreted as suggesting that treatment with the plant extract would reduce plasma glucose levels and the concomitant glucose stress, which the surrounding cells and tissues would have been subjected to. ${ }^{43}$

D. Decreased renal reabsorption of glucose by blocking the sodium-glucose co-transporters (SGLTs). ${ }^{44}$ SGLTs are found predominantly in the intestines, kidneys, and recently in the brain, ${ }^{45}$ where they avidly drive the accumulation of glucose against a concentration gradient, due to the presence of inward sodium electrochemical potential gradients across the cell membrane. ${ }^{46,47}$ The most prevalent and functionally important SGLT in the kidney is SGLT2, where it accounts for $90 \%$ of glucose reabsorption in the kidney and has attracted a great deal of interest in the field of diabetes. The possibility of modulation of the SGLT system via anti-sense molecules or inhibition with antagonists is being evaluated in Phase III clinical trials. It is interesting that the discovery of SGLTs was as targets for Phlorizin, a dihydrochalcone first isolated from the bark of apple trees by French chemists. It was found to induce hypoglycemia in dogs without affecting insulin levels. ${ }^{48}$ The rationale behind anti-diabetes therapy based on SGLT2-inhibition is that by attenuating renal glucose reabsorption, postprandial hyperglycemia can be reduced and the development of diabetes complication prevented or slowed down, without necessarily involving insulin activity. ${ }^{49}$

E. PPAR alpha, PPAR delta, and PPAR gamma agonists: the peroxisome proliferator-activated receptors (PPARs) are lipid-activated transcription factors belonging to the nuclear receptor superfamily, which includes three distinct subtypes - PPAR $\alpha, \operatorname{PPAR} \gamma$, and $\operatorname{PPAR} \delta(\beta)$. Stimulation of PPAR $\alpha$ increases the synthesis of healthy high-density lipoprotein cholesterol (HDL-C), PPAR $\gamma$ plays a critical role in promoting lipid storage, ${ }^{50}$ while PPAR $\delta$ suppresses macrophage-derived inflammation, enhances fatty acid catabolism, and energy uncoupling in adipose tissue and muscle. ${ }^{51}$ These properties suggest that stimulants for the PPARs will increase energy expenditure, enhance weight loss, and therefore enforce desirable anti-diabetic effects. Peroxisome agonists were selected in a HTS using a cell-based trans-activation assay. Hits were defined as compounds that induced luciferase activity in cells transiently cotransfected with luciferase reporter gene plasmid containing the GAL4 binding element and a chimeric plasmid consisting of the yeast GAL4 DNA-binding domain fused to the ligand binding domain of PPARdelta. Specificity for PPARdelta was later determined by testing the promising compounds 
in in vitro assays, where the ligand binding domain in the co-transfected chimeric plasmid originated from PPARalpha, PPARgamma, or the Retinoid X receptor.

F. TGR5 agonists: when activated by bile acids, the G protein-coupled receptor TGR5 drives increased energy expenditure in brown adipose tissue of mice fed a high fat diet. Consistent with the role of TGR5 in promoting the dissipation of energy, is the significant weight gain and mitochondrial dysfunction in skeletal muscle that is observed in female TGR5 knockout mice when challenged with a high fat diet. ${ }^{53}$ Therefore, the discovery of phytochemicals which stimulate TGR5 and hence boost mitochondrial function, would be relevant for preventing and treating T2D. TGR5 agonists were selected using stably transformed TGR5-expressing CHO cells co-transformed with CRE-driven luciferase reporter plasmids. Upon exposure to plant extracts or $10 \mu \mathrm{M}$ lithocholic acid (positive control), positive hits were identified as those with increased luminescence. ${ }^{54}$

In summary, the drug candidates which emerge from the drug discovery process will have promising activity against biological targets of interest, but little will be known about their safety, toxicity, pharmacokinetics, and metabolism in humans. In reality, some of the drugs developed within the last decades failed to make the market due to toxicity and lack of efficacy detected late in the drug discovery process. ${ }^{55}$ The major reason for incurring the high costs of drug development before rejecting the drug candidates comes from the fact that the problems with safety and pharmacokinetics are only detected during clinical trials that are carried out as the last leg in the process.

\section{Finding phytopreparations with innovative and more effective anti-diabetic properties}

As new knowledge is gathered of the molecular basis of diabetes pathophysiology, more bioluminescence-based bioassays like those described above in (E) and (F) can be constructed with any target of interest and used to screen for useful agonists amongst the thousands of plant-derived secondary metabolites. A question that we have been faced with in studying herbals used in traditional medicine to treat diabetes is as follows: when considering an alleged anti-diabetic phytotherapy which does not acutely reduce blood glucose levels, will any or a combination of all of the above bioassays be useful in revealing its mechanism(s) of action?

A case in point is the investigation of the proof of efficacy for the so-called Rauvolfia-Citrus decoction (RC). We have previously shown that the combination of calorie-restriction and chronic (6 weeks) administration of RC to genetic diabetic (BKS- $d b)$ mice resulted in normalization of blood glucose, reduction in lipid contents of the mice eyes, as well as in the protection of the fragile BKS- $d b$ pancreas. ${ }^{56} \mathrm{RC}$ is a decoction used in Nigerian traditional medicine to treat illnesses with diabetes-like symptoms and is prepared from boiling foliage of Rauvolfia vomitoria and fruits of Citrus aurantium. The BKS- $d b$ mice were used here because they mimic human T2D with complications as expressed in their impaired blood circulation, restricted physical activity, fragile pancreas, and poor wound healing. In a randomized, doubleblinded, and placebo-controlled pilot clinical trial, a 4-month administration of RC to Danish T2D patients already stabilized on OADs, led to significant reductions in postprandial plasma glucose and $\mathrm{HbA} 1 \mathrm{c}$ values, without adverse effects or significant weight loss. ${ }^{28}$ Although basal phosphorylated acetyl CoA carboxylase was significantly reduced with RC treatment, basal AMP-activated protein kinase expression was unaffected, suggesting that the treatment does not enhance beta-oxidation via AMPK activity. Neither treatment with $\mathrm{RC}$ tea nor the placebo caused changes in body weight in the study subjects. However, RC-treated individuals showed a tendency to decreasing skeletal muscle triglycerides, while increasing the phospholipid fraction when compared with placebo. Therefore, most of these indications seen with testing $\mathrm{RC}$ in in vivo animal and human experiments would be missed if we had relied on using in vitro bioassays.

Interestingly, in both mice and man, the reductions in blood glucose levels were not acute, but were seen after weeks (with mice) and months (in man) of the treatment, suggesting that the primary point of action of the RC therapy is not in reducing blood glucose. Therefore, in the next section, we advance the arguments already put forward for applying Systems Biology (SB) technologies (pharmacogenomics, proteomics, and metabolomics) to the understanding and prediction of the mechanisms of action of medicinal plants with anti-diabetic potentials in in vivo systems. ${ }^{57-59}$ Because the 'omics methods measure global profiles of genes, proteins, and metabolites as each molecule plays its critical role in the biology of the organism, the SB approach simplifies and economizes the discovery of innovative antidiabetic treatment solutions.

\section{Reductionists versus multi-targeted approaches to drug discovery}

Whereas modern drug development emphasizes the development of a patentable single compound or a "magic bullet" to treat a specific condition, traditional medicine 
often aims to restore balance to the whole organism by using chemically complex materials and/or extracts from different plants, in order to maximize a synergistic effect or to improve the likelihood of the interaction with the intended targets. Insofar as the specific condition under consideration is a single symptom or pathogen, the reductionist approach to drug selection and identification works quite well. However, the evaluation of potential therapeutic agents for complex disorders like diabetes demands quite different approaches. Firstly, diabetes like other chronic and complex diseases is multifactorial in nature. Secondly, humans vary so much in their susceptibility that none of the genes that could predispose people to $\mathrm{T} 2 \mathrm{D}$ has so far been found to be predominant. ${ }^{60-62}$ It is therefore for illnesses like diabetes that the complex mixtures used in traditional medicine come into their own, as they might provide different compounds acting in synergy or pro-drugs which become activated when metabolized.

Therefore, as already proposed by Verpoorte, ${ }^{58}$ we promote the suggestion that plant preparations and phytochemicals derived from medicinal plants pre-selected in traditional medicine be investigated in vivo for their safety and anti-diabetic efficacy using carefully selected disease model animals, or in specially-designed randomized and placebo-controlled clinical trials. The addition advocated here is that, in conjunction with the standard clinical biochemical analyses of blood glucose, lipid, and toxicity measurements, body fluids and tissue samples should be taken and analyzed using a combination of technologies that have been developed in the field of Systems Biology (SB). The research activities proposed for the integrative SB-based drug discovery strategy are illustrated in Figure 2. Apart from furnishing us with a holistic view of the safety and efficacy of the intervention under investigation, the specific outcomes of the SB strategy include provision of evidenced-backed therapeutic agents, molecular modeling of diabetes pathophysiology, savings in time and money for drug development, and identification of the patients' group for whom the treatment solution is best suited.

The SB technologies are large-scale gene, protein, metabolite measurements, commonly referred to as the 'omics technologies (ie, pharmacogenomics, proteomics, and metabolomics, respectively). Although lipids constitute a subset of the metabolite fraction in cells, their unique chemistry and importance in many metabolic diseases such as obesity, atherosclerosis, stroke, hypertension, and

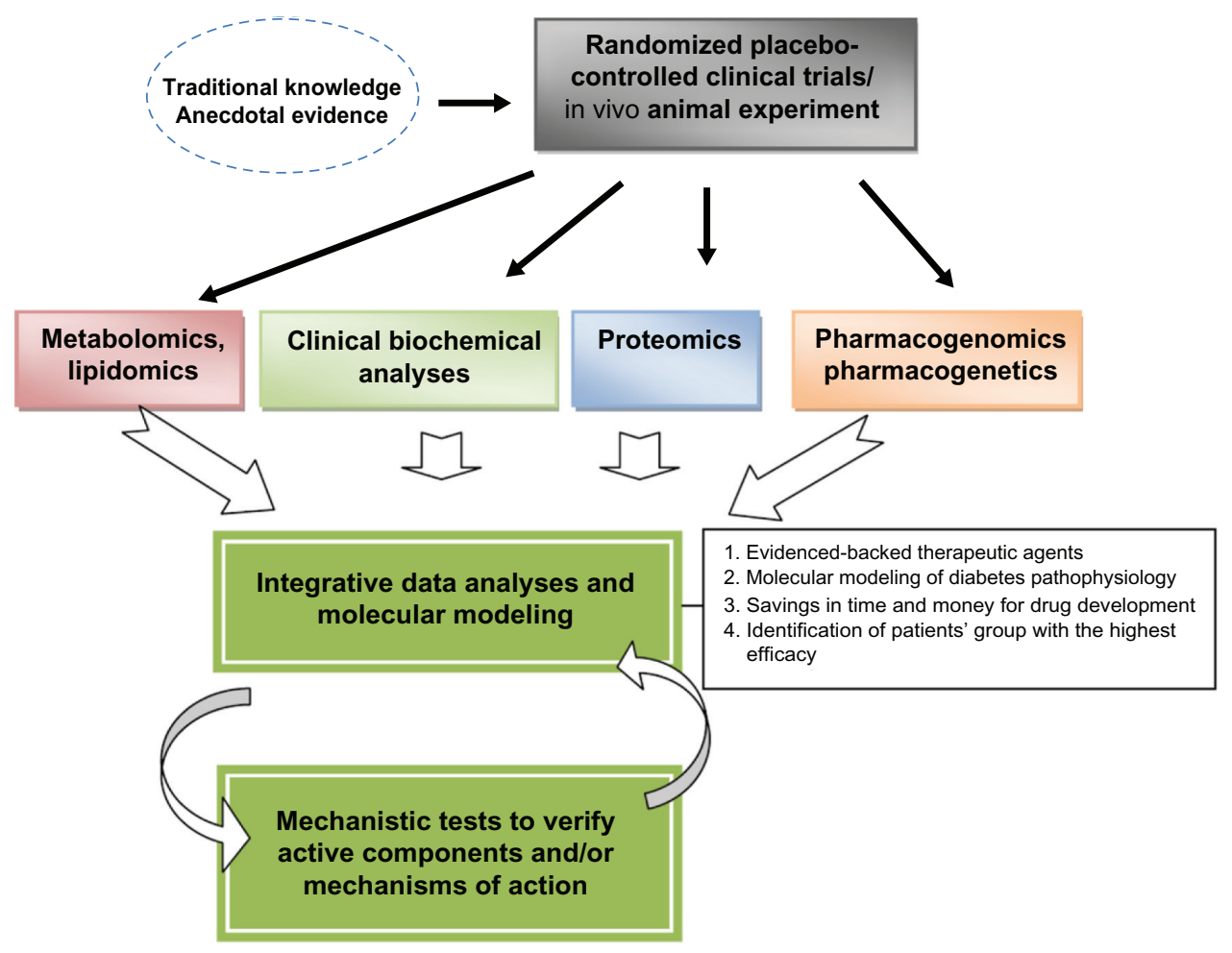

Figure 2 Clinical validation of medicinal plants using systems biology technologies.

Notes: The dark arrows indicate patient samples collected at the clinical trial or at in vivo pre-clinical animal experiments and sent for the different bio-analyses, while the clear arrows indicate data and information flow for integrative data analyses and molecular modeling. Reiterative testing of individual fractions in highly discriminative in vivo model systems or in in vitro bioassays is advocated for reliable knowledge of the mechanisms of action. 
diabetes have driven the development of special technological platforms dedicated to lipidomics or the study of qualitative and quantitative changes in lipid fingerprinting of selected tissues. SB is a rapidly growing multidisciplinary field aimed at describing and understanding complex interactions within biological systems by analyses of the nature and quantities of the constituent macromolecules. Special attention is given to how the interactions give rise to the function and behavior of the entire organism. In the last decade, increased interests in SB technologies coupled with the development of powerful, computer-driven, user-friendly multivariate statistics gave reductions in costs and accelerated studies of fluxes in gene expression and metabolite profiles within the entire organism, as well as changes resulting from interactions between the organism and its environment. Hence the SB approach is now well suited for investigations of the pathophysiology and drug development processes in diabetes, a chronic disease resulting from complex interactions between multiple genetic and environmental factors.

\section{Examples of applications of SB technologies to studying complex systems}

A few examples of studies where the different 'omics studies have already been used for generating accurate and dynamic pictures of the phenotype of complex mammalian systems when subjected to environmental stimuli like exercise, food, or parasites include:

- $\mathrm{VO}_{2 \max }$ training response is genetically determined: Timmons et $\mathrm{al}^{63}$ used RNA expression profiling of skeletal muscle samples to produce a distinct 26-gene set that predicts favorable $\mathrm{VO}_{2 \max }$ training response. Interestingly, the RNA expression levels of the predictor genes (accounting for $\sim 50 \%$ of the total variance for changes in maximal aerobic capacity), were stable to exercise training in both low and high responders. Thus, the largely higher pre-exercise levels of several of the predictor genes found amongst the high responders, appears to give them the greater potential of benefiting from training.

- Gut microbiome assists humans consuming a nutenriched diet: in a randomized, parallel-group feeding trial designed to evaluate the clinical effects of a 12-week mixed nuts-enriched diet (30 g/day) in human subjects with the metabolic syndrome, non-targeted metabolomics strategy revealed unexplored metabolic pathways impacted by the diet. ${ }^{64}$ Amongst the 20 unique chemical markers of nut intake detected in the human urine samples, were glucuronide and sulfate conjugates of urolithin A (dihydroxydibenzopyran-6-one) - the characteristic gut microbial-derived metabolites of walnut hydrolyzable tannins - ellagitannins (ETs). ETs are not absorbed by humans and are not common amongst edible plants, being confined to walnuts, pecans, cashews, hazelnuts, and some berries. However, the microbial transformation of the ETs within the intestinal lumen provides the more absorbable urolithin derivatives. Here is an example of how the gut microbiome influences human health/disease by changing items in diet. As the authors rightly point out, this effect of a mixed nuts diet could not be predicted or detected without using the metabolomics strategy.

- Early detection of Schistosoma japonicum infection using metabolite fluxes in infected mice bio-fluids.

Schistosomiasis is a chronic infectious disease affecting about 600 million people who are exposed to the trematode, schistosome worldwide. Current diagnostic methods are unable to detect schistosomiasis at its early stages, such that the disease is first diagnosed via passage of blood in urine or feces, when the parasite has established in the human host and is causing serious complications in various organs. Upon investigating metabolic fluxes in mice bio-fluids and liver tissues from animals subjected to $S$. japonicum infection, Wu et $\mathrm{al}^{65}$ were able to detect schistosomiasis at the third week post-infection, a week earlier than 'gold standard' methods. S. japonicum infection caused significant elevation of urinary 3 -ureidopropionate, a uracil catabolic product. Furthermore, the changes in 3-ureidopropionate content and overall metabolic changes in both urinary and plasma samples were closely correlated with the time-course of disease progression.

As with other technologies used for investigating biological systems, there is the risk of artifacts, false positives and negatives, as well as several technological challenges that need to be managed and mitigated. Firstly, apart from pharmacogenomics/transcriptomics which provide genomewide representations based on chip technology, none of the other 'omic platforms provide comprehensive coverage of all the metabolites in plasma or muscle samples. In proteomics and metabolomics, the general practice is to either focus on the abundant varieties or target specific groups. Secondly, detecting specific cause-effect relationships between the many RNA, protein, lipid, and metabolite changes generated as a result of physiological adaptations to the environmental stimuli can be daunting. The temptation is to focus on the variance attributable to the stronger signals, even though they may not be the important underlying factors. Therefore, 
no single 'omic approach is comprehensive by itself; we propose that it is the integration and comparison of all the 'omics platforms that brings the added value to applying SB methodology to studying medicinal plants.

\section{Advantages with the SB-based approach to drug development starting from plant materials}

The advantages of the proposed methodology are that:

1. SB approach provides internally consistent explanations of whole body energy homeostasis: nontargeted metabolomics of body fluids of study subjects undergoing placebo-controlled clinical trials have the potential for providing novel biomarkers of the disease, as well as revealing biomarkers of drug efficacy. Contextualization of the metabolomics results is enhanced by integration of study data from the clinical biochemical analyses and the other 'omic platforms, namely pharmacogenomics and lipidomics, thus linking the fluxes in the metabolites and genes to relevant biochemical pathways and processes. Combinations of metabolomics and transcriptomics data have been used to successfully investigate T2D-specific biomarkers in genetically diabetic mice. ${ }^{66}$

2. The components of SB approach serve as internal checks for one another. As each of the 'omics technology is run independent of the other and independent of the routine biochemical analyses, the fluxes in one form of analysis should be consistent with the findings in the others. For example, if our Rauvolfia-Citrus ${ }^{28,56}$ treatment really brings about reduction of skeletal muscle triglycerides in a lipid profile analysis (detected by lipid profiling of skeletal muscle), there ought to be increased expression of skeletal muscle genes involved in lipid breakdown (detected by pharmacogenomics of skeletal muscle), that should also influence plasma lipid levels (detected in routine clinical biochemical analyses).

3. The SB approach results in substantial reductions in drug development costs in time and money as the effects of the different components in the investigational medicine on both desired targets and on off-targets are spotted in the one trial. ${ }^{67}$ This means that the go/no-go decision can be made much earlier in the drug development process. The different compounds within one herbal preparation may provide important combination therapies that simultaneously affect multiple pharmacological targets and provide clinical efficacy well beyond that achievable with single component-based drugs. The verification of the identified contributions from individual components can then be rationalized in specifically designed in vitro and/or in vivo bio-guided assays.

\section{Conclusion}

Most of the plant-derived compounds that have been found to be medicinally useful and interesting tend to be secondary metabolites. Not only have medicinal plants provided raw material for innovative and useful treatment solutions, but the unique biochemistry of the secondary metabolites and their interactions with ligands have provided pointers for (novel) rational drug development that would otherwise not be intuitive to the chemist. The application of the SB approach to investigating drug safety and efficacy within the context of the entire living system can in principle be applied in any drug development process. However, the administration of random synthetic compounds to humans may be dangerous as the study subjects will be exposed to the same safety issues of unacceptable toxicity, responsible for the current crisis facing modern drug development. This is why medicinal plants that have been selected through hundreds of years of empirical findings in traditional medicine are well suited to being investigated with the SB approach. The story of Phlorizin, its effects on the kidney for glucose regulation, and current efforts to modulate that regulatory system via pharmacological means, is a good example of an innovative anti-diabetic treatment based on medicinal plants. Given the increased advantage of investigating medicinal plants for anti-diabetic properties by integrating the 'omics technologies with randomized and placebo-controlled clinical studies, we wish to reiterate with Moerman (cited in Gertsch ${ }^{36}$ ) that although 'the low-hanging fruits (of Ethnopharmacology) have already been picked', the chances are now increased for discovering innovative and more effective phytotherapies. An added advantage of the $\mathrm{SB}$ approach is that the findings will enhance the quality and integrity of affordable traditional medicines that are already within reach in the poorer developing world. The suggested strategy is not intended to imply that current in vitro bioassay-based approaches become redundant. On the contrary, the SB-driven drug discovery approach should only be introduced at the beginning of the drug development process to filter out noise and assist in the identification of those truly novel therapeutic principles. All the fractions/compounds indicated by the SB-'fishing' procedure will need to be validated. In the validation phase, there may be the necessity of designing new in vitro bioassays, but there will also be the need to use some of 
the well-established tests that target different aspects of diabetes pathophysiology.

\section{Disclosure}

The authors report no conflicts of interest in this work.

\section{References}

1. Bailey CJ, Day C. Traditional plant medicines as treatments for diabetes. Diabetes Care. 1989;12:553-564.

2. Beckmann R. Biguanide (Experimenteller Teil). In: Maske H, editor. Oral wirksame Antidiabetika. Vol. 29 of Handbuch der experimentellen Pharmakologie. [(Experimental). In: Maske H, editor. Orally active antidiabetic agents, Vol 29 of Handbook of experimental Pharmacology]. (German) Berlin, Germany: Springer-Verlag; 1971:439-596. (cited in reference 1).

3. Nathan DM, Buse JB, Davidson MB, et al; American Diabetes Association, European Association for Study of Diabetes. Medical management of hyperglycemia in type 2 diabetes: a consensus algorithm for the initiation and adjustment of therapy: a consensus statement of the American Diabetes Association and the European Association for the Study of Diabetes. Diabetes Care. 2009;32:193-203.

4. Danaei G, Finucane MM, Lu Y, et al; Global Burden of Metabolic Risk Factors of Chronic Diseases Collaborating Group (Blood Glucose). National, regional, and global trends in fasting plasma glucose and diabetes prevalence since 1980: systematic analysis of health examination surveys and epidemiological studies with 370 countryyears and 2.7 million participants. Lancet. 2011;378:31-40.

5. Roglic G, Unwin N. Mortality attributable to diabetes: estimates for the year 2010. Diabetes Res Clin Pract. 2010;87:15-19.

6. Shaw JE, Sicree RA, Zimmet PZ. Global estimates of the prevalence of diabetes for 2010 and 2030. Diabetes Res Clin Pract. 2010;87:4-14.

7. International Diabetes Federation. The global burden. Available at: http://www.idf.org/diabetesatlas/5e/the-global-burden. Accessed on May 22, 2012.

8. Nejentsev S, Walker N, Riches D, Egholm M, Todd JA. Rare variants of IFIH1, a gene implicated in antiviral responses, protect against type 1 diabetes. Science. 2009;324:387-389.

9. Silver K, Mitchell BD, Walston J, et al. TRP64 ARG beta 3-adrenergic receptor and obesity in Mexican Americans. Hum Genet. 1997;101: 306-311.

10. Caramori ML, Canani LH, Costa LA, Gross JL. The human peroxisome proliferator-activated receptor $\gamma 2$ (PPAR $\gamma$ ) Pro12Ala polymorphism is associated with decreased risk of diabetic nephropathy in patients with type 2 diabetes. Diabetes. 2003;52:3010-3013.

11. Liu L, Zheng T, Wang F, et al. Pro12Ala polymorphism in the PPARG gene contributes to the development of diabetic nephropathy in Chinese type 2 diabetic patients. Diabetes Care. 2010;33:144-149.

12. Mootha VK, Lindgren CM, Eriksson KF, et al. PGC-1-responsive genes involved in oxidative phosphorylation are coordinately down-regulated in human diabetes. Nat Genet. 2003;34:267-273.

13. Jin T, Liu L. The Wnt signaling pathway effector TCF7L2 and type 2 diabetes mellitus. Mol Endocrinol. 2008;22(11):2383-2392.

14. DeFronzo RA. From the triumvirate to the ominous octet: a new paradigm for type 2 diabetes mellitus [Banting Lecture]. Diabetes. 2009; 58:773-795.

15. DIAMAP. Road Map Report. 2010 Sep. Available at: http://www. euradia.org/eu-projects/diamap. Accessed on May 22, 2012.

16. American Diabetes Association. Standards of Medical Care in Diabetes - 2011. Diabetes Care. 2011;34(S1):S11-S61.

17. Dixon JB, O'Brien PE, Playfair J, et al. Adjustable gastric banding and conventional therapy for type 2 diabetes: a randomized controlled trial. JAMA. 2008;299:316-323.

18. World Health Organization. Traditional medicine fact sheet No 134. Available at: http://www.who.int/mediacentre/factsheets/fs134/en/. Accessed on May 22, 2012.
19. Gurib-Fakim A. Medicinal plants: traditions of yesterday and drugs of tomorrow. Mol Aspects Med. 2006;27:1-93.

20. Bechter AM. The civilizing of curare: a history of its development and introduction into anesthesiology. Anesth Analg. 1977;56(2):305-319.

21. Ritchie JM, Greene NM. Local Anesthetics. In: Gilman AG, Rall TW, Nies AS, et al. Goodman and Gilman's The Pharmacological Basis of Therapeutics, 8th ed. New York: Pergamon Press; 1990:311.

22. Kuruüzüm-Uz A. Editorial. Botanics: Targets and Therapy. 2011;1: $1-4$.

23. Srinivasan K. Role of spices beyond food flavoring: nutraceuticals with multiple health effects. Food Reviews International. 2005;21: 167-188.

24. Yeh GY, Eisenberg DM, Kaptchuk TJ, Phillips RS. Systematic review of herbs and dietary supplements for glycemic control in diabetes. Diabetes Care. 2003;26:1277-1294.

25. Kamble SM, Kamlakar PL, Vaidya S, Bambole VD. Influence of Coccinia indica on certain enzymes in glycolytic and lipolytic pathway in human diabetes. Indian J Med Sci. 1998;52:143-146.

26. Vuksan V, Sievenpiper JL, Koo VY, et al. American ginseng (Panax quinquefolius L.) attenuates postpranidal glycemia in a time-dependent but not dose-dependent manner in healthy individuals. Am J Clin Nutr. 2001;73:753-758.

27. Akilen R, Tsiami A, Devendra D, Robinson N. Glycated haemoglobin and blood pressure-lowering effect of cinnamon in multi-ethnic Type 2 diabetic patients in the UK: a randomized, placebo-controlled, doubleblind clinical trial. Diabet Med. 2010;27(10):1159-1167.

28. Campbell-Tofte JIA, Mølgaard P, Josefsen K, et al. Randomized and double-blinded pilot clinical study of the safety and anti-diabetic efficacy of the Rauvolfia-Citrus tea, as used in Nigerian Traditional Medicine. J Ethnopharmacol. 2011;133:402-411.

29. Vlietinck A, Pieters L, Apers S. Legal requirements for the quality of herbal substances and herbal preparations for the manufacturing of herbal medicinal products in the European Union. Planta Med. 2009; 75:683-688.

30. Marcus D, Grollman A. Botanical medicines: The need for new regulations. N Engl J Med. 2002;347(25):2073-2075.

31. Li S, Han Q, Qiao C, Song J, Cheng CL, Xu H. Chemical markers for the quality control of herbal medicines: An overview. Chin Med. 2008; 28(3):7.

32. Lu Q, Chow SC, Tse SK. Assessing the consistency of traditional Chinese medicine with multiple correlative active components. J Biopharm Stat. 2007;17:791-808.

33. Calixto JB. Efficacy, safety, quality control, marketing and regulatory guidelines for herbal medicines (phytotherapeutic agents). Braz J Med Biol Res. 2000;33:179-189.

34. Sucher NJ, Carles MC. Genome-based approaches to the authentication of medicinal plants. Planta Med. 2008;74(6):603-623.

35. McIntyre M. Protecting the availability of herbal medicines. J Altern Complement Med. 1999;5:109-113.

36. Gertsch J. How scientific is the science in ethnopharmacology? Historical perspectives and epistemological problems. J Ethnopharmacol. 2009;122:177-183.

37. Oubré AY, Carlson TJ, King SR, Reaven GM. From plant to patient: an ethnomedical approach to the identification or new drugs for the treatment of NIDDM. Diabetologia. 1997;40(5):614-617.

38. Houghton PJ, Howes M-J, Lee CC, Steventon G. Uses and abuses of in vitro tests in ethnopharmacology: Visualizing an elephant. J Ethnopharmacol. 2007;110:391-400.

39. Srijayanta S, Jones PM, Persaud S, Lawrence MJ, Raman A. In vitro screening of medicinal plants for potential anti-diabetic effects. $J$ Pharm Pharmacol. 1998;50(S9):219.

40. Persaud SJ, Al-Majed H, Raman A, Jones PM. Gymnema sylvestre stimulates insulin release in vitro by increased membrane permeability. J Endocrinol. 1999;163:207-212.

41. Ali H, Houghton PJ, Soumyanath A. Alpha-Amylase inhibitory activity of some Malaysian plants used to treat diabetes; with particular reference to Phyllanthus amarus. J Ethnopharmacol. 2006;107:449-455. 
42. Guerrero RO, Khan MTH, Casañas B, Morales M. Specific bioassays with selected plants of Bangladesh. Rev Cubana Plant Med. 2004;9(2).

43. Liu F, Kim JK, Li Y, Liu X, Li J, Chen X. An extract of Lagerstroemia speciosa has insulin-like glucose uptake-stimulatory and adipocyte differentiation-inhibitory activities in 3T3-L1 cells. J Nutr. 2001;131: 2242-2247.

44. Chao EC. A paradigm shift in diabetes therapy - dapagliflozin and other SGLT2 inhibitors. Discov Med. 2011;11(58):255-263.

45. Yu AS, Hirayama BA, Timbol G, et al. Functional expression of SGLTs in rat brain. Am J Physiol Cell Physiol. 2010;299(6):C1277-C1284.

46. Diez-Sampedro A, Wright EM, Hirayama BA. Residue 457 controls sugar binding and transport in the $\mathrm{Na}(+)$ /glucose cotransporter. $J$ Biol Chem. 2001;276:49188-49194.

47. Rahmoune H, Thompson PW, Ward JM, Smith CD, Hong G, Brown J. Glucose transporters in human renal proximal tubular cells isolated from the urine of patients with non-insulin-dependent diabetes. Diabetes. 2005;54(12):3427-3434.

48. Ehrenkranz RRL, Lewis NG, Kahn CR, Roth J. Phlorizin, a review. Diabetes Metab Res Rev. 2005;21:31-38.

49. White JR. Apple trees to sodium glucose co-transporter inhibitors: a review of SGLT2 inhibition. Clin Diabetes. 2010;28(1):5-10.

50. Staels B, Fruchart JC. Therapeutic roles of peroxisome proliferatoractivated receptor agonists. Diabetes. 2005;54:2460-2470.

51. Barish GD, Narkar VA, Evans RM. PPAR $\delta$ : A dagger in the heart of the metabolic syndrome. J Clin Invest. 2006;116(3):590-597.

52. Epple R, Cow C, Xie Y, et al. Novel bisaryl substituted thiazoles and oxazoles as highly potent and selective peroxisome proliferatoractivated receptor $\delta$ agonists. J Med Chem. 2010;53:77-105.

53. Watanabe M, Houten SM, Mataki C, et al. Bile acids induce energy expenditure by promoting intracellular thyroid hormone activation. Nature. 2006;439:484-489.

54. Sato H, Genet C, Strehle A, et al. Anti-hyperglycemic activity of a TGR5 agonist isolated from Olea europaea. Biochem Biophys Res Commun. 2007;362:793-798.

55. Kola I, Landis J. Can the pharmaceutical industry reduce attrition rates? Nat Rev Drug Discov. 2004;3:711-716.

56. Campbell JIA, Mortensen A, Mølgaard P. Tissue lipid lowering-effect of a traditional Nigerian anti-diabetic infusion of Rauwolfia vomitoria foliage and Citrus aurantium fruit. J Ethnopharmacol. 2006;104: 379-386.

57. Wang M, Lamers RJ, Korthout HA, et al. Metabolomics in the context of systems biology: bridging traditional Chinese Medicine and molecular pharmacology. Phytother Res. 2005;19:173-182.

58. Verpoorte R, Choi YH, Kim HK. Ethnopharmacology and systems biology: a perfect holistic match. J Ethnopharmacol. 2005;100: 53-56.

59. Ulrich-Merzenich G, Zeitler H, Jobst D, Panek D, Vetter H, Wagner H. Application of the "-omic-" technologies in phytomedicine. Phytomedicine. 2007;14:70-82.

60. Gloyn AL, Weedon MN, Owen KR, et al. Large-Scale association studies of variants in genes encoding the pancreatic $\beta$-Cell KATP channel subunits Kir6.2 (KCNJ11) and SUR1 (ABCC8) confirm that the KCNJ11 E23K variant is associated with type 2 diabetes. Diabetes. 2003;52(2):568-572.
61. Scott LJ, Mohlke KL, Bonnycastle LL, et al. A genome-wide association study of Type 2 Diabetes in Finns detects multiple susceptibility variants. Science. 2007;316(5829):1341-1345.

62. Lango H; UK Type 2 Diabetes Genetics Consortium, Palmer CN, Morris AD, Zeggini E, Hattersley AT, McCarthy MI, et al. Assessing the combined impact of 18 common genetic variants of modest effect sizes on Type 2 Diabetes risk. Diabetes. 2008;57(11):3129-3135.

63. Timmons JA, Knudsen S, Rankinen T, et al. Using molecular classification to predict gains in maximal aerobic capacity following endurance exercise training in humans. J Appl Physiol. 2010;108: 1487-1496.

64. Tulipani S, Llorach R, Jáuregui O, et al. Metabolomics unveils urinary changes in subjects with metabolic syndrome following 12-week nut consumption. J Proteome Res. 2011;10(11):5047-5058.

65. Wu J, Xu W, Ming Z, Dong H, Tang H, Wang Y. Metabolic changes reveal the development of schistosomiasis in mice. PLoS Negl Trop Dis. 2010;4(8):e807.

66. Connor SC, Michael K, Hansen MK, et al. Integration of metabolomics and transcriptomics data to aid biomarker discovery in type 2 diabetes. Mol Biosyst. 2010;6:909-921.

67. Schmidt BM, Ribnicky DM, Lipsky PE, Raskin I. Revisiting the ancient concept of botanical therapeutics. Nat Chem Biol. 2007;3:360-366.

68. Jia W, Gao W, Tang L. Antidiabetic herbal drugs officially approved in China. Phytotherapy Research. 2003;17:1127-1134.

69. Atawodi SE. Antioxidant potential of African medicinal plants. Afr J Biotechnol. 2005;4:128-133.

70. Bnouham M, Ziyyat A, Mekhf H, Tahri A, Legssye A. Medicinal plants with potential anti-diabetic activity - A review of ten years of herbal medicine research (1990-2000). Int J Diabetes Metab. 2006; 14:1-25.

71. Jung M, Park M, Lee HC, Kang YH, Kang ES, Kim SK. Anti-diabetic agents from medicinal plants. Curr Med Chem. 2006;13:1203-1218.

72. Khalil NM, Pepato MT, Brunetti IL. Free radical scavenging profile and myeloperoxidase inhibition of extracts from anti-diabetic plants: Bauhinia forficata and Cissus sicyoides. Biol Res. 2008;41: 165-171.

73. Evans JL. Preclinical and clinical evidence for botanical interventions for Type 2 Diabetes. In: Cooper R, Kronenberg F, editors. Botanical Medicine: From Bench to Bedside. Santa Clara, CA: Mary Ann Liebert, Inc Publishers; 2009:13-32.

74. Afolayan AJ, Sunmonu TO. In vivo studies on antidiabetic plants used in South African herbal medicine. J Clin Biochem Nutr. 2010; 47:98-106.

75. Begum R, Mosihuzzaman M, Azad Khan AK, Nahar N, Liaquat A. Emerging challenge of Type 2 Diabetes: Prospects for Medicinal Plants, recent advances in the pathogenesis, prevention and management of type 2 diabetes and its complications, Mark B Ximering, ISBN: 978-953-307-597-6. Publisher, InTech. (DOI: 10.5772/20704).

76. Kavishankar GB, Lakshmidevi N, Mahadeva Murthy S. Diabetes and medicinal plants-A review. Int J Pharm Biomed Science. 2011;2(3): 65-68.

77. Patel DK, Prasad SK, Kumar R, Hemalatha S. An overview on antidiabetic medicinal plants having insulin mimetic property. Asian Pac J Trop Biomed. 2012;4:320-330.
Botanics: Targets and Therapy

\section{Publish your work in this journal}

Botanics: Targets and Therapy is an international, peer-reviewed, open access journal focusing on the discovery and development of active compounds based upon or found naturally occurring in the plant kingdom that may have therapeutic potential in any disease state. The manuscript management system is completely online and includes a very

quick and fair peer-review system. Visit http://www.dovepress.com/ testimonials.php to read real quotes from published authors.

\section{Dovepress}

Submit your manuscript here: http://www.dovepress.com/botanics-targets-and-therapy-journa 